hep-th/9708002

MRI-PHY/P970720

Revised

\title{
Strong Coupling Dynamics of Branes from M-theory
}

\author{
Ashoke Sen'尹 \\ Mehta Research Institute of \\ Mathematics and Mathematical Physics \\ Chhatnag Road, Jhusi, Allahabad 221506, INDIA
}

\begin{abstract}
We study some aspects of the strong coupling dynamics of Dirichlet six branes, antisix branes, and orientifold planes by using the equivalence of type IIA string theory and $M$-theory on $S^{1}$. In the strong coupling limit there exists static configuration of brane and anti-brane at arbitrary separation, suspended in an external magnetic field. The mass of the open string stretched between the brane and the anti-brane approaches a finite positive value even when the branes coincide. Similar result is obtained for a Dirichlet six brane on top of an orientifold six plane. We also derive the anomalous gravitational interaction on the brane and the orientifold plane from $M$-theory.
\end{abstract}

\footnotetext{
${ }^{1}$ On leave of absence from Tata Institute of Fundamental Research, Homi Bhabha Road, Bombay 400005, INDIA

${ }^{2}$ E-mail: sen@mri.ernet.in, sen@theory.tifr.res.in
} 


\section{Introduction and Summary}

Many known properties of Dirichlet six branes in type IIA string theory [1, 2, 3] have been reproduced [4, 5, 6, 7] by using the known identification [8]-[19] of these branes with Kaluza-Klein monopoles[20, 21] in $M$-theory. In this paper we shall use this identification to derive some properties of these D-branes which cannot be derived using the standard string perturbation theory.

The first system we shall analyse is the brane - anti-brane configuration. This corresponds to a complicated interacting system in perturbative string theory due to the appearance of tachyonic open string states for sufficiently small separation between the brane and the anti-brane 22, 23, 24]. However, by mapping it to the known Kaluza-Klein dipole solution 21, 25 in $M$-theory, we show that the dynamics simplifies in the strong coupling limit of string theory. The dipole solution in eleven dimensional supergravity theory can be interpreted as a static brane - anti-brane configuration in type IIA string theory, suspended in an external magnetic field[26]. We calculate the mass of the open string stretched between the brane and the anti-brane by identifying it with the $M$-theory membrane stretched along the bolt of the Kaluza-Klein dipole solution. While this answer agrees with the expected answer from string theory for large separation between the branes, it approaches a finite value proportional to $m_{\text {string }} g_{\text {string }}$ when the separation between the brane and the anti-brane vanishes. In perturbative string theory, the classical contribution to the mass vanishes in this limit, and quantum fluctuations on the open string makes this into a tachyonic state 22.

The second system that we shall analyze will be a D- six brane in the presence of an orientifold six plane 11, 27]. For this we use the identification of the orientifold plane with the Atiyah-Hitchin space of $M$-theory [28, 7]. In string perturbation theory, the classical mass of the open string, stretched between the D- six brane and its image, is proportional to the distance between the D-brane and the orientifold plane. When the D-brane is on top of the orientifold plane, the classical mass vanishes, but the massless states get projected out, and the lowest mass state from this sector has mass of order unity in the string scale. From the $M$-theory viewpoint, on the other hand, this configuration of coincident D-brane and orientifold plane is described by the double cover of the AtiyahHitchin space 28], and the open string stretched between the D-brane and its image is represented by a membrane wrapped around the bolt of the Atiyah-Hitchin space. The 
classical mass of this state is proportional to the area of the bolt, and when converted to the string scale, this mass again turns out to be proportional to $m_{\text {string }} g_{\text {string }}$. This is an exact result in the strong coupling limit of string theory.

In the final section, we shall deviate somewhat from the main theme of the paper, and use the $M$-theory - IIA correspondence to derive a known result, - the anomalous gravitational coupling of the six brane. This result was derived in refs. 29, 30] by other methods. We derive this by starting from the $C \wedge X_{8}$ coupling in $M$-theory [31, 32, and integrating it over the transverse space of the six brane in $M$-theory. The same method can be used to prove the existence of a similar coupling on the orientifold plane. This has been derived using different method in a recent paper by Dasgupta, Jatkar and Mukhi[33].

\section{Brane - Anti-brane Configuration}

Our starting point for studying the brane - anti-brane configuration will be the KaluzaKlein dipole solution given in [21]. The solution embedded in eleven dimensions is described by the metric:

$$
\begin{aligned}
d s^{2}= & -d t^{2}+\sum_{m=5}^{10} d y^{m} d y^{m}+\left(r^{2}-a^{2} \cos ^{2} \theta\right)\left[\Delta^{-1} d r^{2}+d \theta^{2}\right] \\
& +\left(r^{2}-a^{2} \cos ^{2} \theta\right)^{-1}\left[\Delta\left(d x^{4}+a \sin ^{2} \theta d \psi\right)^{2}+\sin ^{2} \theta\left(\left(r^{2}-a^{2}\right) d \psi-a d x^{4}\right)^{2}\right],
\end{aligned}
$$

where,

$$
\Delta=r^{2}-2 M r-a^{2} .
$$

$M$ and $a$ are parameters labelling the solution. Let us define,

$$
\kappa=\frac{\sqrt{M^{2}+a^{2}}}{2 M\left(M+\sqrt{M^{2}+a^{2}}\right)}, \quad \Omega=\kappa \frac{a}{\sqrt{M^{2}+a^{2}}},
$$

and

$$
\phi=\psi-\Omega x^{4} .
$$

The solution (1) is regular if $r \geq r_{0}$ where,

$$
r_{0}=M+\sqrt{M^{2}+a^{2}},
$$

and $x^{4}$ and $\phi$ have periodicities $2 \pi / \kappa$ and $2 \pi$ respectively. If we use $x^{4}$ and $\phi$ as independent angular coordinates, then the surface $r=r_{0}$ is a fixed point of the killing vector 
$\partial / \partial x^{4}$ and represents a bolt. (1) represents a valid solution of $M$-theory in the limit of large $M$, when the curvature associated with the solution is small, and hence the higher derivative terms in the Lagrangian are not important.

In appropriate coordinate system, this describes a magnetic monopole - anti-monopole pair suspended in an external magnetic field[26]. In order to see this, we shall first analyze this solution for $a>>M$. First of all, note that in the region $(r-a)>>M, \Delta$ can be approximated as:

$$
\Delta \simeq\left(r^{2}-a^{2}\right)
$$

Substituting this into the metric (1), and defining new coordinates $\rho$ and $\bar{\theta}$ through the relations:

$$
\rho \sin \bar{\theta}=\sqrt{r^{2}-a^{2}} \sin \theta, \quad \rho \cos \bar{\theta}=r \cos \theta,
$$

we can bring the metric (1) into the form

$$
d s^{2}=-d t^{2}+\sum_{m=5}^{10} d y^{m} d y^{m}+\left(d x^{4}\right)^{2}+d \rho^{2}+\rho^{2}\left(d \bar{\theta}^{2}+\sin ^{2} \bar{\theta} d \psi^{2}\right) .
$$

This appears to be the standard flat metric on $R^{9,1} \times S^{1}$. However the background is non-trivial because of the twisted boundary condition on $x^{4}$ and $\psi$ (as $x^{4}$ gets translated by $2 \pi / \kappa, \psi$ must get translated by $2 \pi \Omega / \kappa$ in order to have an identification of points in space-time). There are many ways of choosing independent angular coordinates - one of them being $x^{4}$ and $\phi$ defined in eq.(俄 - but we shall take the independent coordinates to be $x^{4}$ and

$$
\widetilde{\phi}=\phi+\kappa x^{4}=\psi+(\kappa-\Omega) x^{4}
$$

The asymptotic space-time may then be interpreted as $M$-theory compactified on $S^{1}$ labelled by $x^{4}$, in the presence of a magnetic flux 34, 35, 36]. The magnetic field on the $z$ axis is given by:

$$
B=\Omega-\kappa \simeq-\frac{M}{4 a^{2}} \quad \text { for } \quad a>>M .
$$

We shall now turn to the interior region where $(r-a)$ is of order $M$. In order to properly interpret the solution, we also consider the single monopole solution 20, 21]:

$$
\begin{gathered}
d s^{2}=-d t^{2}+\sum_{m=5}^{10} d y^{m} d y^{m}+d s_{T N}^{2}, \\
d s_{T N}^{2}=\left(1+\frac{4 m}{r}\right) d \vec{r}^{2}+\left(1+\frac{4 m}{r}\right)^{-1}\left(d x^{4}+4 m(1-\cos \theta) d \phi\right)^{2},
\end{gathered}
$$


where $x^{4}$ has periodicity $16 \pi m$. This represents a Kaluza-Klein monopole of magnetic charge $4 m$. We can interpret both the monopole and dipole solution as solutions in the same theory by matching the $x^{4}$ periodicity in the two theories. This gives,

$$
m=\frac{1}{8 \kappa} .
$$

We shall now show that the solution (11) corresponds to a monopole-antimonopole pair, separated by a distance $2 a$ for large $a$. This will be done by showing that i) in the limit of large $a$, the metric around $r=r_{0}, \theta=0(\theta=\pi)$ reduces to that of a Kaluza-Klein anti-monopole (monopole), and ii) for finite $a$, but close to the point $r=r_{0}, \theta=0(\theta=\pi)$ the metric agrees with the metric close to a Kaluza-Klein anti-monopole (monopole). For this we introduce a new set of coordinates:

$$
\left(r_{0}-M\right) \sin ^{2} \theta=\widetilde{\rho}(1-\cos \tilde{\theta}), \quad 2\left(r-r_{0}\right)=\widetilde{\rho}(1+\cos \widetilde{\theta})
$$

First we focus on the region near $\left(r=r_{0}, \theta=0\right)$ and take the $a \rightarrow \infty$ limit keeping $\left(r-r_{0}, \theta \sqrt{a}\right)$ finite. In this limit, the metric (11) reduces to:

$$
\begin{aligned}
d s^{2}= & -d t^{2}+\sum_{m=5}^{10} d y^{m} d y^{m}+\left(1+\frac{M}{\widetilde{\rho}}\right)^{-1}\left(d x^{4}-M(1-\cos \widetilde{\theta}) d \widetilde{\phi}\right)^{2} \\
& +\left(1+\frac{M}{\widetilde{\rho}}\right)\left(d \widetilde{\rho}^{2}+\widetilde{\rho}^{2} d \widetilde{\theta}^{2}+\widetilde{\rho}^{2} \sin ^{2} \widetilde{\theta} d \widetilde{\phi}^{2}\right)
\end{aligned}
$$

The coordinate ranges are $0 \leq \widetilde{\rho}<\infty, 0 \leq \widetilde{\theta} \leq \pi$. Furthermore, $x^{4}$ and $\widetilde{\phi}$ can be taken to be independent angular coordinates with periods $4 \pi M$ and $2 \pi$ respectively. Thus we see that (15) represents the metric of a Kaluza-Klein anti-monopole with magnetic charge $-M$. Similar analysis can be carried out near $\left(r=r_{0}, \theta=\pi\right)$.

On the other hand if we keep a finite and examine the solution close to the region $\widetilde{\rho}=0$, the metric reduces to:

$$
\begin{aligned}
d s^{2}= & -d t^{2}+\sum_{m=5}^{10} d y^{m} d y^{m}+(2 \kappa \widetilde{\rho})\left(d x^{4}-(2 \kappa)^{-1}(1-\cos \widetilde{\theta}) d \widetilde{\phi}\right)^{2} \\
& +(2 \kappa \widetilde{\rho})^{-1}\left(d \widetilde{\rho}^{2}+\widetilde{\rho}^{2} d \widetilde{\theta}^{2}+\widetilde{\rho}^{2} \sin ^{2} \widetilde{\theta} d \widetilde{\phi}^{2}\right)
\end{aligned}
$$

Using eq.(13), we can recognise it as the metric given in eq.(11), (12) close to the KaluzaKlein anti-monopole. This is a reflection of the fact that in the choice of coordinates we have made, the metric near $r=r_{0}, \theta=0$ represents an anti- self-dual NUT[25]. Similarly, the metric near $r=r_{0}, \theta=\pi$ represents a self-dual NUT and hence a magnetic monopole. 
As has already been stated, the Kaluza-Klein monopoles in $M$-theory can be interpreted as D- six branes of type IIA string theory 99. Thus the solution (1) describes a static D6-brane - anti- D6-brane configuration of type IIA string theory. The distance between the brane and the anti-brane can be defined to be the geodesic distance between the points $\left(r_{0}, \theta=0\right)$ and $\left(r_{0}, \theta=\pi\right)$. This is given by

$$
l=\int_{0}^{\pi} \sqrt{r_{0}^{2}-a^{2} \cos ^{2} \theta} d \theta \simeq 2 a \quad \text { for } \quad a>>M
$$

Existence of brane anti-brane solution at arbitrary separation $l$ (parametrized by $a$ ) shows that the static force between the brane and the anti-brane vanishes. Since the gravitational and electromagnetic interaction between a brane and an anti-brane are both attractive[3, 22, 23, 24, there is a net attractive force between them. This is cancelled by the repulsive force between the brane and the antibrane, induced by the external magnetic field. For monopole and anti-monopole carrying magnetic charges $\pm M$ and separated by a distance $2 a$, this repulsive force is equal to $-2 M B \simeq M^{2} / 2 a^{2}$ for large $a$. On the other hand, the magnetic attraction betwen this pair is $M^{2} / 4 a^{2}$. The gravitational (and scalar induced) attraction is equal in magnitude to the magnetic attraction. Thus we see that the net attractive force cancels the net repulsive force, giving rise to a static configuration.

We shall now identify the open string state stretched between the brane and the antibrane, and calculate its classical mass. In analogy with the results of ref. [7] this state must be represented by an $M$-theory membrane wrapped on a suitable two cycle. For the dipole solution, such a two cycle is provided by the surface $r=r_{0}$, which appears as a bolt if we choose $\phi$ and $x^{4}$ as the independent angular coordinates. $\theta$ and $\phi$ are good coordinates on the bolt, and the metric on the bolt is given by:

$$
d s_{B}^{2}=\left(r_{0}^{2}-a^{2} \cos ^{2} \theta\right)^{-1}\left(r_{0}^{2}-a^{2}\right)^{2} \sin ^{2} \theta d \phi^{2}+\left(r_{0}^{2}-a^{2} \cos ^{2} \theta\right) d \theta^{2} .
$$

The area of this surface in this metric is given by:

$$
A=4 \pi\left(r_{0}^{2}-a^{2}\right)=8 \pi M\left(M+\sqrt{M^{2}+a^{2}}\right) .
$$

For large $a$, this reduces to $8 \pi M a$, which is simply the product of the period $4 \pi M$ of $x^{4}$, and the separation $2 a$ between the brane - anti-brane pair. If $T_{M}$ denotes the membrane tension in $M$ theory then this state has mass $T_{M} A$. Since $T_{M}$ times the period of the $x^{4}$ direction can be identified as the string tension of type IIA theory, we see that for large 
separation between the branes, the mass of the string is given by the product of the string tension and the separation, - as expected from perturbative string analysis.

In fact, not only is the mass formula reproduced correctly for large $a$, but the shape of the membrane also has the right form so as to be interpreted as a string of length $2 a$. To see this, let us note from (18) that for fixed $\theta$, the radius of the $\phi$ direction is given by:

$$
\frac{\left(r_{0}^{2}-a^{2}\right) \sin \theta}{\sqrt{r_{0}^{2}-a^{2} \cos ^{2} \theta}} \int_{0}^{2 \pi} d \phi \simeq 4 \pi M \quad \text { for large } a, \quad \theta \neq 0, \pi .
$$

Thus away from the north and the south pole $(\theta=0, \pi)$ the bolt has the shape of a cylinder of radius $4 \pi M$. As seen from (1), the compact direction on the cylinder is spanned by $\phi=\widetilde{\phi}+\kappa x^{4}$ at fixed value of $x^{4}+a \sin ^{2} \theta \psi$. For large $a$ and finite $\theta$, this means that the compact direction is spanned by $x^{4}$ at fixed $\tilde{\phi}$. The length of this cylinder, stretched along the $\theta$ direction, is equal to $l$ defined in (17). A membrane wrapped around such a surface clearly looks like an elementary type IIA string stretched from $\theta=0$ to $\theta=\pi$, covering a distance $2 a$.

When $a$ is of the order of $M$, the ten dimensional interpretation is not reliable, reflecting the fact that the magnetic field required to hold the brane - anti-brane pair apart produces curvature in the non-compact direction which is of the same order as the size of the compact direction [26, 34, 35, 36]. Note, however, that the solution is completely nonsingular in eleven dimensions. For $a=0$, we again have a ten dimensional interpretation of the solution if we take $\phi$ instead of $\widetilde{\phi}$, and $x^{4}$ as the independent angular coordinates. In this case the asymptotic space has the structure of $R^{9,1} \times S^{1}$ with flat metric. Furthermore the metric does not have any $g_{4 \phi}$ component, showing that the solution has zero magnetic field. Thus it is natural to interpret this configuration as a coincident brane-anti-brane pair. The alert reader may raise several objections to this proposal. First of all, note that if we continue to use $\widetilde{\phi}$ and not $\phi$ as the azimuthal coordinate of the transverse space, then the solution for $a=0$ can be interpreted as a monopole - anti-monopole pair at the two poles of the surface $r=r_{0}$ 26]. From (17) we see that the separation $l$ remains finite even for $a=0$. Note, however, that when the brane-anti-brane separation $a$ is of the order of their internal size, the notion of distance between them becomes ambiguous, as the presence of the brane (and the anti-brane) significantly modifies the ambient metric. Thus $l$ is not a good measure of the brane anti-brane separation in this range of parameters. A more appropriate measure of the brane anti-brane separation might be the strength of the magnetic field that is required to hold them apart. As seen from (10), if we continue 
to use $\widetilde{\phi}$ as the azimuthal angle, then the magnetic field $B$ does reach a finite value as $a \rightarrow 0$. Furthermore, if we reduce $a$ further by making it negative, keeping $\kappa$ fixed, the magnetic field continues to increase in magnitude. This might be taken as an evidence for further shrinkage of the brane - anti-brane separation. However, from (11), it is clear that in $M$-theory configurations with positive and negative $a$ are related to each other by a simple coordinate transformation. Thus even if we interprete the negative $a$ values as a description of smaller brane - anti-brane separation, we reach the conclusion that this configuration is equivalent to one with a larger brane - anti-brane separation. From this point of view, the $a=0$ solution represents the minimum possible separation between the brane and the anti-brane, - in the same sense that the self-dual radius represents the minimum radius of compactification of a string theory - as long as the force that holds them apart is induced by an external magnetic field. Whether we call this a configuration of coincident brane - anti-brane pair or not is a matter of convention. Due to the fact that precisely at this point there is a choice of coordinate system that makes the solution free from any magnetic field, we choose to call it coincident brane - anti-brane pair.

When the separation between the brane and the anti-brane vanishes, the classical mass of an open string stretched between them goes to zero in perturbative string theory. The quantum contribution to the mass will be of the order of the string scale and makes this into a tachyonic state [22, 23, 24]. In contrast, in the $M$-theoretic description, the area of the bolt, as given in (19), reduces to a finite non-zero answer $16 \pi M^{2}$ for $a=0$. Noting that for $a=0$ the radius of the $x^{4}$ direction is $R=4 M$, we see that this mass is given by $\pi R^{2}$. It is instructive to express this mass in the conventional string units. For this we note that so far in our calculation we have set the eleven dimensional plank scale to unity, but now we can explicitly put in that scale. In this case the mass of the wrapped membrane is proportional to $m_{p}^{3} R^{2}$, where $m_{p}$ denotes the eleven dimensional Plank mass. By standard duality chasing, we can express this in string units 37. If $m_{S}$ denotes the string mass scale, and $g_{S}$ the string coupling constant in ten dimensions, then the relevant relations are:

$$
m_{p}^{3} R=m_{S}^{2}, \quad g_{S}=\left(m_{p} R\right)^{3 / 2} .
$$

Using these relations we see that for $a=0$ the mass of the wrapped membrane is proportional to $m_{S} g_{S}$.

Finally we note that even though the solution does not suffer from the instability due to tachyonic open string modes stretched between the D-branes, there are other instabilities 
in the solution. In particular, since it represents a brane - anti-brane pair balanced precariously in a magnetic field, a slight relative displacement of the pair, without an accompanying change in the magnetic field, will throw the configuration off balance[26]. This, however, represents a known physical phenomenon, and does not correspond to any new feature of the brane - anti-brane interaction.

\section{Brane on an Orientifold Plane}

An orientifold six plane of type IIA string theory is described in $M$-theory[28, 7] by the Atiyah-Hitchin metric 38, 39]:

$$
\begin{gathered}
d s^{2}=-d t^{2}+\sum_{m=5}^{10} d y^{m} d y^{m}+(8 m)^{2} d s_{A H}^{2}, \\
d s_{A H}^{2}=f(\rho)^{2} d \rho^{2}+a(\rho)^{2} \sigma_{1}^{2}+b(\rho)^{2} \sigma_{2}^{2}+c(\rho)^{2} \sigma_{3}^{2},
\end{gathered}
$$

where $f, a, b$ and $c$ are functions defined in ref. 39,

$$
\rho=r / 8 m
$$

and,

$$
\begin{aligned}
\sigma_{1} & =-\sin \psi d \theta+\cos \psi \sin \theta d \phi \\
\sigma_{2} & =\cos \psi d \theta+\sin \psi \sin \theta d \phi, \\
\sigma_{3} & =d \psi+\cos \theta d \phi .
\end{aligned}
$$

The coordinate $x^{4}$ is related to $\psi$ as:

$$
x^{4}=16 m \psi .
$$

The coordinate ranges are given by $\pi \leq \rho<\infty, 0 \leq \theta \leq \pi, \phi$ is periodic with period $2 \pi$, and $\psi$ is periodic with period $2 \pi$. Finally, there are two identifications under the transformations $I_{1}$ and $I_{3}$ given by:

$$
\begin{gathered}
I_{1}: \quad(\rho, \theta, \phi, \psi) \rightarrow(\rho, \pi-\theta, \pi+\phi,-\psi), \\
I_{3}: \quad(\rho, \theta, \phi, \psi) \rightarrow(\rho, \theta, \phi, \psi+\pi) .
\end{gathered}
$$

Our interest will be in a configuration of an orientifold plane, together with a D- six brane on top of it. According to ref.[28], the $M$-theory background describing this configuration 
is simply the double cover $\overline{\mathcal{N}}$ of the Atiyah-Hitchin space, which is obtained by modding out the space (23) by the transformation $I_{1}$, but not by $I_{3}$. In order that the asymptotic radius of the $x^{4}$ direction still remains equal to $16 \pi m$, we need to modify eqs.(22), (24) and (26) to

$$
\begin{gathered}
d s^{2}=-d t^{2}+\sum_{m=5}^{10} d y^{m} d y^{m}+(4 m)^{2} d s_{A H}^{2}, \\
\rho=r / 4 m, \quad x^{4}=8 m \psi .
\end{gathered}
$$

This description is valid in the strong coupling limit, when the size $m$ of the manifold is large compared to the eleven dimensional Planck scale.

The space $\overline{\mathcal{N}}$ admits an anti-self-dual harmonic two form $\Omega$, given by [40, 41, 42]:

$$
\Omega=F(\rho)\left(d \sigma_{1}-\frac{f a}{b c} d \rho \wedge \sigma_{1}\right),
$$

where

$$
F(\rho)=F_{0} \exp \left(-\int_{\pi}^{\rho} \frac{f a}{b c} d \rho^{\prime}\right) .
$$

$F_{0}$ is a constant. Thus we can define a $U(1)$ gauge field on the world volume of this system by decomposing the three form field $C$ of $M$-theory as

$$
C(t, y, x)=A(t, y) \wedge \Omega .
$$

This gauge field $A$ can be identified with the $S O(2)$ gauge field living on the world volume of the D-brane - orientifold plane system. Note that $\Omega$ is odd under $I_{3}$ and hence is projected out on $\mathcal{N}$. This explains why there is no gauge field living on an isolated orientifold plane.

We now address the fate of the open string stretched between the D- six brane and its image. In type IIA string theory, the classical contribution to this mass vanishes when the D-brane coincides with the orientifold plane, but there is an oscillator contribution of the order of the string scale. Clearly in $M$-theory this state should be described by a membrane wrapped on an appropriate two cycle. In this case the most obvious choice is again the bolt described by the surface $\rho=\pi$ in the space $\overline{\mathcal{N}}$. The correct coordinate system around the bolt are the new angular coordinates $\widetilde{\theta}, \widetilde{\phi}, \widetilde{\psi}$, and the shifted radial coordinate $\widetilde{\rho}$ defined through the relations:

$$
\begin{aligned}
\widetilde{\rho} & =\rho-\pi \\
\sigma_{2} & =-\sin \widetilde{\psi} d \widetilde{\theta}+\cos \widetilde{\psi} \sin \tilde{\theta} d \widetilde{\phi}
\end{aligned}
$$




$$
\begin{aligned}
\sigma_{3} & =\cos \widetilde{\psi} d \tilde{\theta}+\sin \widetilde{\psi} \sin \tilde{\theta} d \widetilde{\phi} \\
\sigma_{1} & =d \widetilde{\psi}+\cos \widetilde{\theta} d \widetilde{\phi}
\end{aligned}
$$

In this coordinate system, the metric near the bolt takes the form

$$
d s_{A H}^{2} \simeq d \widetilde{\rho}^{2}+4 \widetilde{\rho}^{2}(d \widetilde{\psi}+\cos \widetilde{\theta} d \widetilde{\phi})^{2}+\pi^{2}\left(d \widetilde{\theta}^{2}+\sin ^{2} \theta d \widetilde{\phi}^{2}\right)
$$

The identification under $I_{1}$ implies that $\widetilde{\psi}$ has period $\pi$. Thus the metric is non-singular at $\widetilde{\rho}=0$ and represents the product of a plane and a sphere. The area of the sphere spanned by $\theta$ and $\phi$, after being rescaled by $(4 m)^{2}$ as is required by eq.(29), is given by:

$$
A=64 \pi^{3} m^{2}
$$

Since the radius $R$ of the fourth direction is given by $8 m$, we see that the area is proportional to $R^{2}$. A membrane wrapped around this sphere will have a mass proportional to $m_{p}^{3} R^{2}$. Transformed to the string variables, this would again correspond to a mass of order $g_{S} m_{S}$.

In order to verify that the membrane state that we have considered really represents the open string state that we are looking for, note that near the bolt, $\Omega$ is given by,

$$
\Omega \simeq F_{0}\left(\sin \tilde{\theta} d \tilde{\phi} \wedge d \widetilde{\theta}-\frac{2}{\pi^{2}} \widetilde{\rho} d \widetilde{\rho} \wedge(d \widetilde{\psi}+\cos \tilde{\theta} d \widetilde{\phi})\right)
$$

From this we see that the integral of $\Omega$ on the bolt is non-zero. Thus the wrapped membrane state is charged under the gauge field $A$ living on the D-brane world volume. This is precisely what is expected of an open string stretched from the D-brane to its image.

\section{Anomalous Gravitational Coupling of the D-brane and the Orientifold Plane}

It has been known for sometime 29, 30 that a D- six brane in general background has an anomalous interaction term of the form:

$$
\int C \wedge p_{1}
$$

where $C$ and $p_{1}$ are the pull back of the three form gauge field and the pontrjagin density from the space-time to the world volume. In this section we shall derive this by representing the six brane as the Kaluza-Klein monopole of $M$-theory. 
In $M$-theory, there is an anomalous interaction term of the form [32]:

$$
\int C \wedge X_{8}(R),
$$

where $X_{8}$ is an appropriate eight form constructed out of the curvature tensor. We shall now evaluate this on an eleven dimensional space of the form:

$$
(T N) \times K_{7},
$$

where $(T N)$ denotes the Euclidean self-dual Taub-NUT space and $K_{7}$ is a seven dimensional space with Minkowski signature. Using the identification of the Euclidean TaubNUT space with the transverse space of the six-brane, we can interpret (40) as a six brane with world volume wrapped on $K_{7}$. Since $X_{8}$ contains a term proportional to $p_{1} \wedge p_{1}$, and since

$$
\int_{T N} p_{1}=-2,
$$

(39), evaluated in such a background gives a term of the form:

$$
a \int_{K_{7}} C \wedge p_{1}
$$

where $a$ is a constant.

This is precisely the term that we wanted. It remains to verify that the coefficient $a$ is given correctly, but before we do that, let us note that a similar coupling between the 3 -form field $C$ and the curved orientifold six plane 33] can be derived by representing the transverse space of the orientifold plane by the Atiyah-Hitchin space. If $L_{7}$ denotes the world-volume of the orientifold plane, we shall get a coupling of the form:

$$
b \int_{L_{7}} C \wedge p_{1}
$$

where the constant $b$ is proportional to the integral of $p_{1}$ on the Atiyah-Hitchin space.

We shall now show that this procedure yields the correct values of the constants $a$ and $b$. To do this, let us first note that since a D- six brane on top of an orientifold plane is represented by the double cover of the Atiyah-Hitchin space, we have the result:

$$
\int_{T N} p_{1}+\int_{A H} p_{1}=2 \int_{A H} p_{1} .
$$

Thus the integral of the pontrjagin index over the Taub-NUT space and the AtiyahHitchin space gives the same answer. This gives $a=b$, as was found in ref.[33] for the orientifold six plane. 
The overall normalization is fixed by noting that $\int_{T N} p_{1}$ given in (41) is $(1 / 24)$ times $\int_{K 3} p_{1}$. Thus $a$ and $b$ are $(1 / 24)$ times the coefficient of the $\int C \wedge p_{1}$ term for $M$-theory on K3. To see that this is the correct answer, consider type IIA of $T^{3} /(-1)^{F_{L}} \Omega \mathcal{I}_{3}$, where $\mathcal{I}_{3}$ denotes the reversal of sign of all the three coordinates on the torus, $(-1)^{F_{L}}$ changes the sign of Ramond sector states on the left, and $\Omega$ is the world-sheet parity transformation. This theory has 16 D-branes and 8 orientifold planes, so the net contribution to the $C \wedge p_{1}$ term has a coefficient equal to $24 a$, i.e. equal to that of K3. But since this theory is dual to $M$-theory on $\mathrm{K} 3$, this is precisely the correct answer.

Acknowledgement: I wish to thank D. Jatkar and S. Mukhi for useful discussions.

\section{References}

[1] J. Dai. R. Leigh and J. Polchinski, Mod. Phys. Lett. A4 (1989) 2073;

R. Leigh, Mod. Phys. Lett. A4 (1989) 2767;

J. Polchinski, Phys. Rev. D50 (1994) 6041;

E. Gimon and J. Polchinski, Phys. Rev. D54 (1996) 1667 [hep-th/9601038].

[2] P. Horava, Nucl. Phys. B327 (1989) 461;

M. Green, Phys. Lett. B266 (1991) 325; Phys. Lett. B329 (1994) 435; Phys. Lett. B354 (1995) 271.

[3] J. Polchinski, Phys. Rev. Lett. 75 (1995) 4724 hep-th/9510017.

[4] P. Ruback, Comm. Math. Phys. 107 (1986).

[5] A. Sen, hep-th/9705212, hep-th/9707042.

[6] Y. Imamura, hep-th/9706144.

[7] A. Sen, hep-th/9707123.

[8] S. Han and I. Koh, Phys. Rev. D31 (1985) 2503;

M. Duff and J. Lu, Nucl. Phys. B416 (1994) 301 hep-th/9306052].

[9] P. Townsend, Phys. Lett. B350 (1995) 184 hep-th/9501068].

[10] M. Costa and G. Papadopoulos, hep-th/9612204]. 
[11] E. Witten, hep-th/9703166].

[12] C. Hull, hep-th/9705162].

[13] E. Bergshoeff, B. Janssen and T. Ortin, hep-th/9706117.

[14] A. Losev, G. Moore and S. Shatashvili, hep-th/9707250.

[15] I. Brunner and A. Karch, [hep-th/9707259].

[16] D. Berenstein, R. Corrado and J. Distler, [hep-th/9704087;

S. Elizur, A. Giveon, D. Kutasov and E. Rabinovici, hep-th/9707217.

[17] A. Hanany and G. Lifschytz, hep-th/9708037.

[18] N. Seiberg and S. Sethi, [hep-th/9708085].

[19] R. Gregory, J. Harvey and G. Moore, hep-th/9708086.

[20] R. Sorkin, Phys. Rev. Lett. 51 (1983) 87.

[21] D. Gross and M. Perry, Nucl. Phys. B226 (1983) 29.

[22] T. Banks and L. Susskind, hhep-th/9511194.

[23] M. Green and M. Gutperle, Nucl. Phys. B476 (1996) 484 hep-th/9604091.

[24] G. Lifschytz, Phys. Lett. B388 (1996) 720 hep-th/9604156;

V. Periwal, hep-th/9612215.

[25] S. Hawking, Phys. Lett. 60A (1977) 81;

G. Gibbons and S. Hawking, Comm. Math. Phys. 66 (1979) 291.

[26] F. Dowker, J. Gauntlett, G. Gibbons and G. Horowitz, Phys. Rev. D53 (1996) 7115 hep-th/9512154.

[27] A. Sagnotti, in Cargese '87, Non-perturbative Quantum Field Theory, ed. G. Mack et. al. (Pergamon Press, 1988) 521;

G. Pradisi and A. Sagnotti, Phys. Lett. B216 (1989) 59;

M. Bianchi, G. Pradisi and A. Sagnotti, Nucl. Phys. B376 (1992) 365;

C. Angelantonj, M. Bianchi, G. Pradisi, A. Sagnotti, and Y. Stanev, Phys. Lett. B385 (1996) 96 hep-th/9606169]; Phys. Lett. B387 (1996) 743 hep-th/9607229. 
[28] N. Seiberg and E. Witten, hep-th/9607163;

N. Seiberg, Phys. Lett. B384 (1996) 81 [hep-th/9606017].

[29] M. Bershadsky, V. Sadov and C. Vafa, Nucl. Phys. B463 (1996) 420 hepth/9511222.

[30] M. Green, J. Harvey and G. Moore, Class. Quant. Grav. 14 (1997) 47 hepth/9605033.

[31] C. Vafa and E. Witten, Nucl. Phys. B447 (1995) 261 hep-th/9505053.

[32] M. Duff, J. Liu and R. Minasian, Nucl. Phys. B452 (1995) 261 hep-th/9506126].

[33] K. Dasgupta, D. Jatkar and S. Mukhi, hep-th/9707224].

[34] F. Dowker, J. Gauntlett, G. Gibbons and G. Horowitz, Phys. Rev. D52 (1995) 6929.

[35] G. Gibbons and K. Maeda, Nucl. Phys. B298 (1988) 741.

[36] F. Dowker, J. Gauntlett, D. Kastor and J. Traschen, Phys. Rev. D49 (1994) 2909;

F. Dowker, J. Gauntlett, S. Giddings and G. Horowitz, Phys. Rev. D50 (1994) 2662.

[37] E. Witten, Nucl. Phys. B443 (1995) 85 hep-th/9503124.

[38] M. Atiyah and N. Hitchin, Phys. Lett. 107A (1985) 21; Phil. Trans. R. Soc. Lond. A315 (1985) 459; The Geometry and Dynamics of Magnetic Monopoles, Princeton Univ. Press (1988).

[39] G. Gibbons and N. Manton, Nucl. Phys. B274 (1986) 183.

[40] G. Gibbons and P. Ruback, Comm. Math. Phys. 115 (1988) 267.

[41] N. Manton and B. Schroers, Ann. Phys. (NY) 225 (1993) 290.

[42] A. Sen, Phys. Lett. B329 (1994) 217 hep-th/9402032. 